\title{
Chemiluminescent Nanomicelles for Imaging Hydrogen Peroxide and Self-Therapy in Photodynamic Therapy
}

\author{
Rui Chen, ${ }^{1}$ Luzhong Zhang, ${ }^{1}$ Jian Gao, ${ }^{2}$ Wei Wu, ${ }^{1}$ Yong Hu, ${ }^{2}$ and Xiqun Jiang1 \\ ${ }^{1}$ Laboratory of Mesoscopic Chemistry and Department of Polymer Science and Engineering, \\ College of Chemistry and Chemical Engineering, Nanjing University, Nanjing 210093, China \\ ${ }^{2}$ National Laboratory of Solid State Microstructure and Department of Material Science and Engineering, \\ Nanjing University, Nanjing 210093, China \\ Correspondence should be addressed to Yong Hu, hvyong@nju.edu.cn and Xiqun Jiang, jiangx@nju.edu.cn
}

Received 20 January 2011; Revised 18 March 2011; Accepted 24 March 2011

Academic Editor: Zhen Cheng

Copyright ( $\odot 2011$ Rui Chen et al. This is an open access article distributed under the Creative Commons Attribution License, which permits unrestricted use, distribution, and reproduction in any medium, provided the original work is properly cited.

Hydrogen peroxide is a signal molecule of the tumor, and its overproduction makes a higher concentration in tumor tissue compared to normal tissue. Based on the fact that peroxalates can make chemiluminescence with a high efficiency in the presence of hydrogen peroxide, we developed nanomicelles composed of peroxalate ester oligomers and fluorescent dyes, called peroxalate nanomicelles (POMs), which could image hydrogen peroxide with high sensitivity and stability. The potential application of the POMs in photodynamic therapy (PDT) for cancer was also investigated. It was found that the PDT-drug-loaded POMs were sensitive to hydrogen peroxide, and the PDT drug could be stimulated by the chemiluminescence from the reaction between POMs and hydrogen peroxide, which carried on a self-therapy of the tumor without the additional laser light resource.

\section{Introduction}

Reactive oxygen species (ROS), a vital substance in organisms, not only involved in many signaling pathways that maintain cellular homeostasis in physiological processes [1] but also can induce severe damage in turn which is called oxidative injury [2]. Hydrogen peroxide $\left(\mathrm{H}_{2} \mathrm{O}_{2}\right)$, one of the ROS, produced in cellular mitochondria, plays a major role in mediating cell growth and apoptosis [3]. In healthy organs, cells produce necessary $\mathrm{H}_{2} \mathrm{O}_{2}$ to mediate diverse physiological progresses. However, abnormal overproduction implicates the development of various pathological conditions, even severe diseases, such as cancer. Moreover, its concentration spans four orders of magnitude from $10^{-8} \mathrm{M}$ in proliferation to $10^{-4} \mathrm{M}$ in apoptosis [4]. Therefore, hydrogen peroxide is a very important signaling molecule acted as an intracellular indicator in the development of cancer [5].

Recently, the detection of hydrogen peroxide in vitro or in vivo has been received much interests [6-8]. By using fluorescence probe techniques, the concentration of hydrogen peroxide can be obtained through fluorescence intensity, helping us to understand the state of cells under physiological and pathological conditions [9]. Another way is peroxalate chemiluminescence (POCL) whose mechanism can be summarized in two progresses [10]. First, peroxalate derivatives can be oxidized only by hydrogen peroxide to generate a high-energy and unstable intermediate: dioxetanedione; then, the intermediate degrades to carbon dioxide as well as transfers energy to nearby molecules, such as fluorescent dye, to give a particular fluorescence $[11,12]$. Using such a feature, a new type of imaging $\mathrm{H}_{2} \mathrm{O}_{2}$ is offered as reported like peroxalate polymeric nanoparticles [13]. These nanoparticles have attractive advantages beyond fluorescence probe, such as tunable wavelength emission, excellent sensitivity, and high specificity for $\mathrm{H}_{2} \mathrm{O}_{2}$, so they can be widely applied in the diagnosis of the diseases related to $\mathrm{H}_{2} \mathrm{O}_{2}$ [14]. However, a major barrier to POCL-based systems application is that peroxalate derivatives are not so stable and inclined to hydrolyze in aqueous or protic solvents [15]. Therefore, the protection of peroxalate esters from hydrolysis is the key factor to peroxalate NPs, for example, the formation of minisize organic reactor in aqueous systems through copolymer 
micelles [16] or microemulsions [17]. Meanwhile, with the peroxalate esters and the fluorescent dyes together in the same phase, the energy transfer is expected to be improved [18].

Photodynamic therapy (PDT) is a new technology for cancer treatment. Photodynamic agents can be excited by laser with specific wavelength, transfer energy to the nearby oxygen, generate strong active singlet oxygen molecules. Then singlet oxygen causes microvascular acute injury, and blood vessel blockage in tumor and induces tumor cells apoptosis, achieving the purpose of local treatment [19]. However, the conventional photodynamic therapy in cancer treatment faces many problems [20]. These photodynamic drugs are hydrophobic and can only dissolve in an organic solvent, which is difficultly to be directly injected into the body. Conventional excitation laser light, ranging from $600 \mathrm{~nm}$ to $700 \mathrm{~nm}$, cannot reach tumor site deeper than $10 \mathrm{~mm}$ from the skin. In addition, in the photodynamic treatment, the tumor localization must be accurately distinguished, because excitation light should only irradiate the tumor tissue, without damaging the normal organs [21]. Therefore, the general photodynamic therapy can only treat superficial tumors, limiting its clinical applications.

In this paper, a novel PDT method is developed by integrating peroxalate ester oligomers, fluorescent dyes, and photodynamic drugs simultaneously inside the PEG-PCL micelles. The hydrophobic core of PEG-PCL micelles can protect the peroxalate ester oligomer from directly contacting the water and postpone their hydrolysis. And the hydrophilic PEG shell outside the PEG-PCL micelles stabilizes these micelles in the aqueous solution, giving them long circulation in the blood, which makes them suitable to be used in biomedical field. The energy between the peroxalate and $\mathrm{H}_{2} \mathrm{O}_{2}$ reaction is effectively transferred to the fluorescent dyes within a confined space, and then dyes release the photon energy for PDT. Within the same micelle, photodynamic drugs easily get the chemiluminescence to generate active singlet oxygen molecules to kill cancer cells. Further, photodynamic drugs encapsulated inside peroxalate nanomicelles (POMs) not only improve their solubility in aqueous solution, but also can be delivered to the tumor site and increase the drug concentration inside the tumor due to the small size of POMs [22]. All can improve the effect of photodynamic therapy with the PDT drug-loaded POMs. Since the excitation is endogenous and does not require external laser light source, this inner "light stick" can be applied in PDT wherever inside the body, no matter how the tumor is deep or close to the skin. The coencapsulated fluorescent dye as a light source for a photosensitizing PDT drug is feasible [23], and intraparticle energy transfer and fluorescence photoconversion are theoretically and experimentally valid [24]. Evidence exists that the apoptosis of tumor cell mainly depends on the content of singlet oxygen, rather than that of hydrogen peroxide [25]. The presence of excess hydrogen peroxide is not only toxic to the normal tissues and cells, but also can induce a malignant tumor. It is very important to reduce the content of hydrogen peroxide and increase the singlet oxygen concentration in the tumor site. Therefore, these PDT drugs-loaded POMs are valuable on the function that not only effectively consumes the hydrogen peroxide in the tumor site, but also transfers the $\mathrm{H}_{2} \mathrm{O}_{2}$ to singlet oxygen through the PDT method. It should be a partial solution to the shortcoming of PDT method such as the short laser wavelength and difficulty in reaching the internal tumor inside the body and have a potential application in photodynamic therapy field.

\section{Materials and Methods}

2.1. Materials. Methoxypolyethyleneglycol (MPEG, Mw: $2 \mathrm{kDa}$, Fluka) was dried in vacuum at $85^{\circ} \mathrm{C}$ for 4 hours before use. E-caprolactone (CL, Sigma) was dehydrated by $\mathrm{CaH}_{2}$ overnight at room temperature and distilled under reduced pressure. Stannous octoate (Sigma) is used as received. Oxalyl chloride (Acros) was distilled under reduced pressure before use. 4-Hydroxybenzyl alcohol (Alfa Aesar), 1, 8-octanediol (Alfa Aesar), 9, 10-diphenyl anthracene (ACROS), rubrene (ACROS), rhodamine B (ACROS), mesotetraphenylporphine (TPP, $\geq 97 \%$, Sterm), and aqueous hydrogen peroxide $\left(\mathrm{H}_{2} \mathrm{O}_{2}, 30 \%\right.$, Sinopharm Chemical Reagent Co. $)$ were used as received. All the other chemicals were of analytical grade and were used without further purification.

\subsection{Synthesis of Poly(ethyleneglycol)-poly(caprolactone)} (PEG-PCL) and Peroxalate Ester Oligomer. PEG-PCL was synthesized by a classical ring-opening polymerization method. Briefly, a small amount of stannous octoate $(0.1 \%$ $\mathrm{w}_{\mathrm{t}} / \mathrm{w}_{\mathrm{t}}$ ) was added into a polymerization tube containing MPEG (3 g, $1.5 \mathrm{mmol})$ and CL monomer $(9.3 \mathrm{~mL})$. The tube was sealed off after being evacuated and then immersed in oil bath at $130^{\circ} \mathrm{C}$ for 48 hours. Then the crude polymerization product was dissolved in $\mathrm{CH}_{2} \mathrm{Cl}_{2}$ and precipitated in an excess mixture of n-hexane and ethyl (v/v:4/1) and then filtered and redissolved in $\mathrm{CH}_{2} \mathrm{Cl}_{2}$. After concentrated with a rotary evaporator, the final product was dried in vacuum. Molecular weight of copolymer was determined by a gel permeation chromatography (GPC, Waters 515 systems) equipped with Waters 525 pump, Waters 2487 Ultraviolet absorbance Detector, and Wyatt Technology Optilab rEX refractive index detector and an STYRAGEL HR3, HR4, and HR5 $(300 \times 7.8 \mathrm{~mm})$ columns in tetrahydrofuran (THF) solution. The poly-dispersity index was calculated based on polystyrene standards with molecular weights in the range from 900 to $1.74 \times 10^{6} \mathrm{~g} / \mathrm{mol}$.

Peroxalate ester oligomer was synthesized with 4-hydroxybenzyl alcohol (16 mmol), 1, 8-octanediol $(2.4 \mathrm{mmol})$ and oxalyl chloride $(18.3 \mathrm{mmol})$. These two kinds of dialcohol were first dissolved in dry tetrahydrofuran (THF, $10 \mathrm{~mL}$ ), with the addition of triethylamine $(40 \mathrm{mmol})$ by an injector under vacuum. The mixture was kept at $0^{\circ} \mathrm{C}$ and then added to oxalyl chloride in dry tetrahydrofuran $(20 \mathrm{~mL})$ protected by a nitrogen atmosphere. The reaction was kept at room temperature overnight and quenched with a saturated brine solution. The product was extracted with ethyl acetate and the combined organic layers were dried by anhydrous $\mathrm{Na}_{2} \mathrm{SO}_{4}$ over 8 hours. After concentrated under vacuum, it was isolated by precipitating in excess 


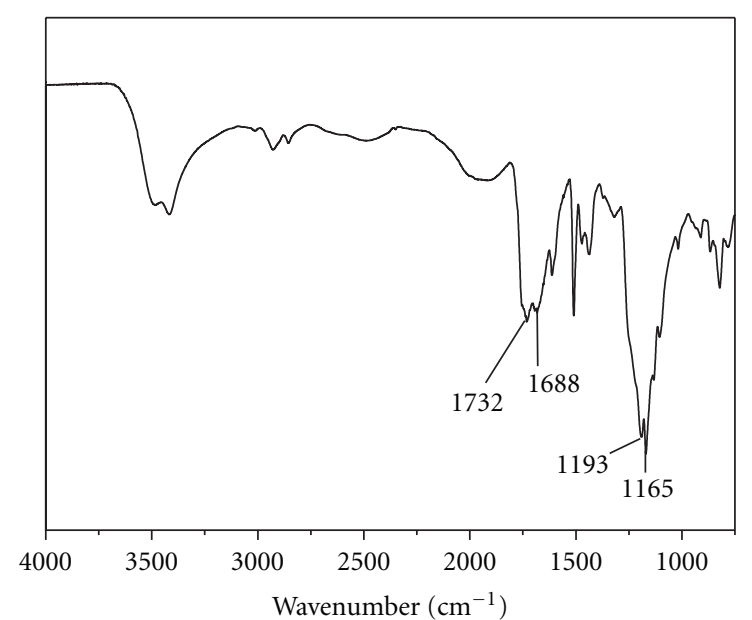

(a)

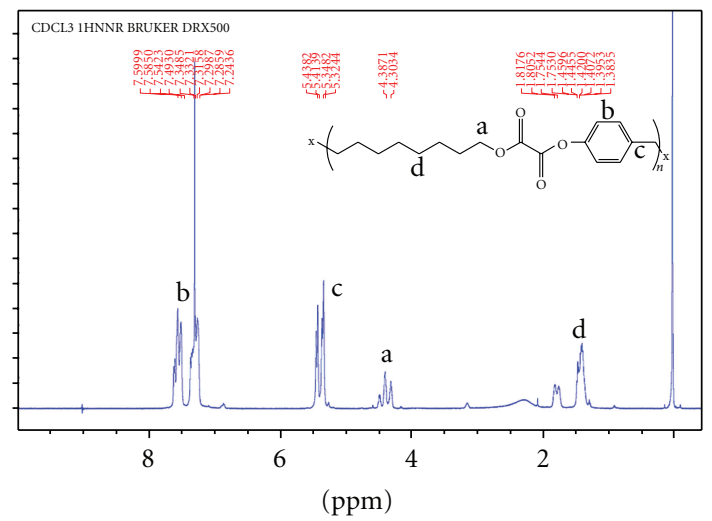

(b)

Figure 1: (a) FT-IR and (b) ${ }^{1} \mathrm{H}-\mathrm{NMR}$ spectra of peroxalate ester oligomers.

dichloromethane/hexane (v/v:1/1) solution, then filtered, and dried in vacuum. The chemical structure of the product was measured with FT-IR (Bruker, Vector 22) using the solid product directly and ${ }^{1} \mathrm{H}-\mathrm{NMR}$ (Bruker DQX-300) in $\mathrm{CDCl}_{3}$ solution.

2.3. Preparation of Chemiluminescent Nanomicelles. The nanomicelles were achieved by the self-assemble block copolymers through a cosolvent evaporation method [16]. PEGPCL $(50 \mathrm{mg})$, peroxalate esters oligomer $(5 \mathrm{mg})$, and the fluorescent dye rubrene $(1 \mathrm{mg})$ were first dissolved in $2 \mathrm{~mL}$ of acetone. The mixture was added dropwise into deionized water $(20 \mathrm{~mL})$ to form micelles with magnetic stirring. Then the organic solvent was removed under reduced pressure at $0^{\circ} \mathrm{C}$, and the resulting micelle solution was used for further experiments.

The hydrodynamic diameter and size distribution of the as-prepared micelles were determined by dynamic light scattering (DLS) using a Brookhaven BI-9000AT system (Brookhaven Instruments Corporation, USA) at a concentration of $1 \mathrm{mg} / \mathrm{mL}$ in deionized water. Morphology of these micelles was conducted on transmission electron microscopy (TEM, JEM-100S, JEOL, Japan).
2.4. Chemiluminescent Characterization of POMs. The nanomicelles solution was used directly with a final concentration of $2.5 \mathrm{mg} / \mathrm{mL}$ in deionized water. The images of chemiluminescence produced by POMs and different concentration of hydrogen peroxide solution (in phosphate buffer $\mathrm{pH}$ 7.4, $0.1 \mathrm{M})$ in a 96-well plate were caught by an imaging system equipped with a $-29^{\circ} \mathrm{C}$ CCD (EC3, UVP). And the mean density of chemiluminescence was statistical by the software. The chemiluminescent intensity of POMs and different concentration of hydrogen peroxide solution (in phosphate buffer $\mathrm{pH} 7.4,0.1 \mathrm{M}$ ) were measured using a luminometer (YN FG-1, Xunjie Corporation, China) and each with three times measurements. Chemiluminescent emission spectrum of peroxalate ester oligomers $(5 \mathrm{mg})$ and dyes $(1 \mathrm{mg})$ in dimethyl phthalate $(2 \mathrm{~mL})$ with hydrogen peroxide $(200 \mu \mathrm{L}, 2 \mu \mathrm{M})$ was measured using a fluorometer spectrofluorometer (RF-5301-PC, Shimadzu) with a turn-off lamp. The stability of POMs in aqueous system was investigated by incubating them in deionized water for various time and then mixed with $0.2 \mu \mathrm{M}$ hydrogen peroxide solution (in phosphate buffer $\mathrm{pH} 7.4,0.1 \mathrm{M}$ ). The chemiluminescent intensity was subsequently measured for 100 seconds using a luminometer (YN FG-1, Xunjie Corporation, China), and the integrated areas under CL intensity curves were calculated as the value.

2.5. Preparation of TPP-Encapsulated POMs. Mesotetraphenylporphine (TPP) was a kind of drug used in photodynamic therapy (PDT). TPP-encapsulated POMs were prepared using the same method as described above with TPP (1 mg) dissolved in acetone. The size of TPP-encapsulated POMs was also investigated by DLS. The chemiluminescence intensity of nanomicelles with TPP or without TPP was measured using a luminometer, at the same time, the influence of TPP to the CL of POMs was also observed.

2.6. The Antitumor Properties of TPP-Encapsulated POMs. Cytotoxicity of TPP-loaded nanomicelles against two kinds of cancer cell lines was assessed by 3-(4, 5-dimethylthiazolyl2)-2, 5-diphenyltetrazolium bromide (MTT) assay. $100 \mu \mathrm{L}$ cells with a density around 5,000 cells/well were seeded in 96-well plate and were grown in completed media having 90\% Dulbecco's Modified Eagle Media (DMEM), 10\% fetal bovine serum, and $100 \mathrm{units} / \mathrm{mL}$ penicillin/streptomycin. The cells were maintained at $37^{\circ} \mathrm{C}$ under $5 \% \mathrm{CO}_{2}$ for 24 hours, then different POMs groups and $\mathrm{H}_{2} \mathrm{O}_{2}$ were added into the cultured media and incubated for another 24 hours. Cytotoxicity with or without $\mathrm{H}_{2} \mathrm{O}_{2}$-treated cells was compared in each drug group. The control cells and only $\mathrm{H}_{2} \mathrm{O}_{2}$-treated cells were also cultured under the same condition. MTT in phosphate buffer saline (PBS) was added into each well and incubated for 4 hours after the media was refreshed. Then the solution was discarded and dimethyl sulphoxide (DMSO, $150 \mu \mathrm{L}$ ) was added into the wells. The optical intensity was measured at $570 \mathrm{~nm}$ using microtiter plate reader. Data were expressed as an average of three times. 


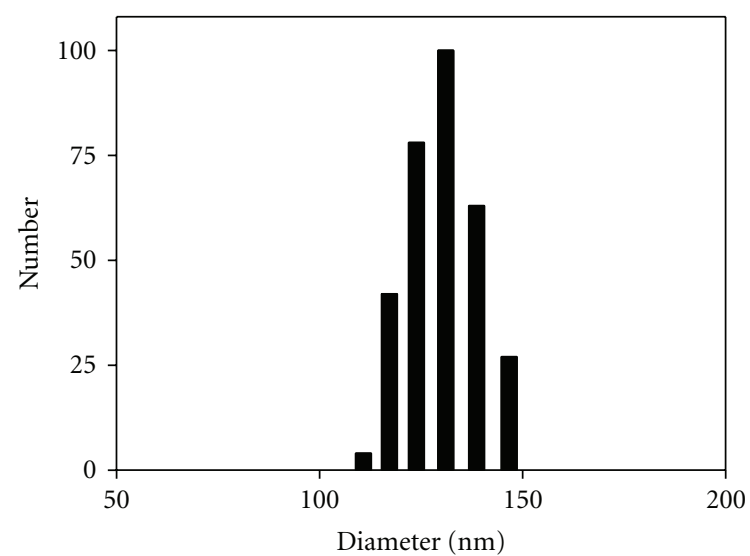

(a)

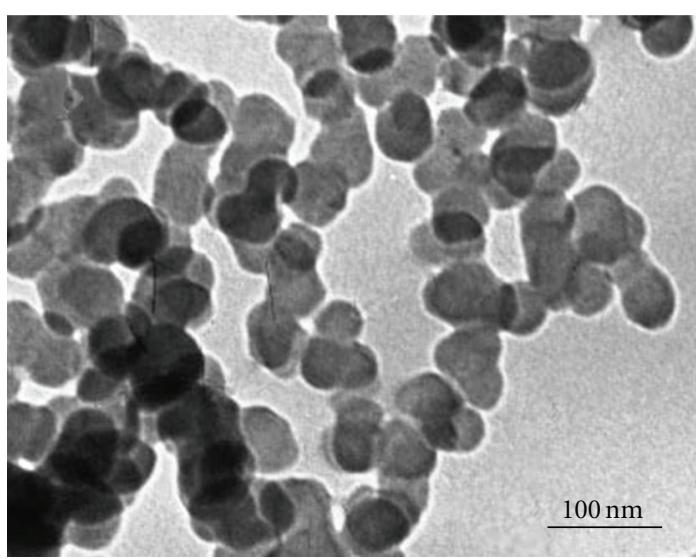

(b)

FIGURE 2: (a) Hydrodynamic diameter distribution and (b) TEM image of the nanomicelles.
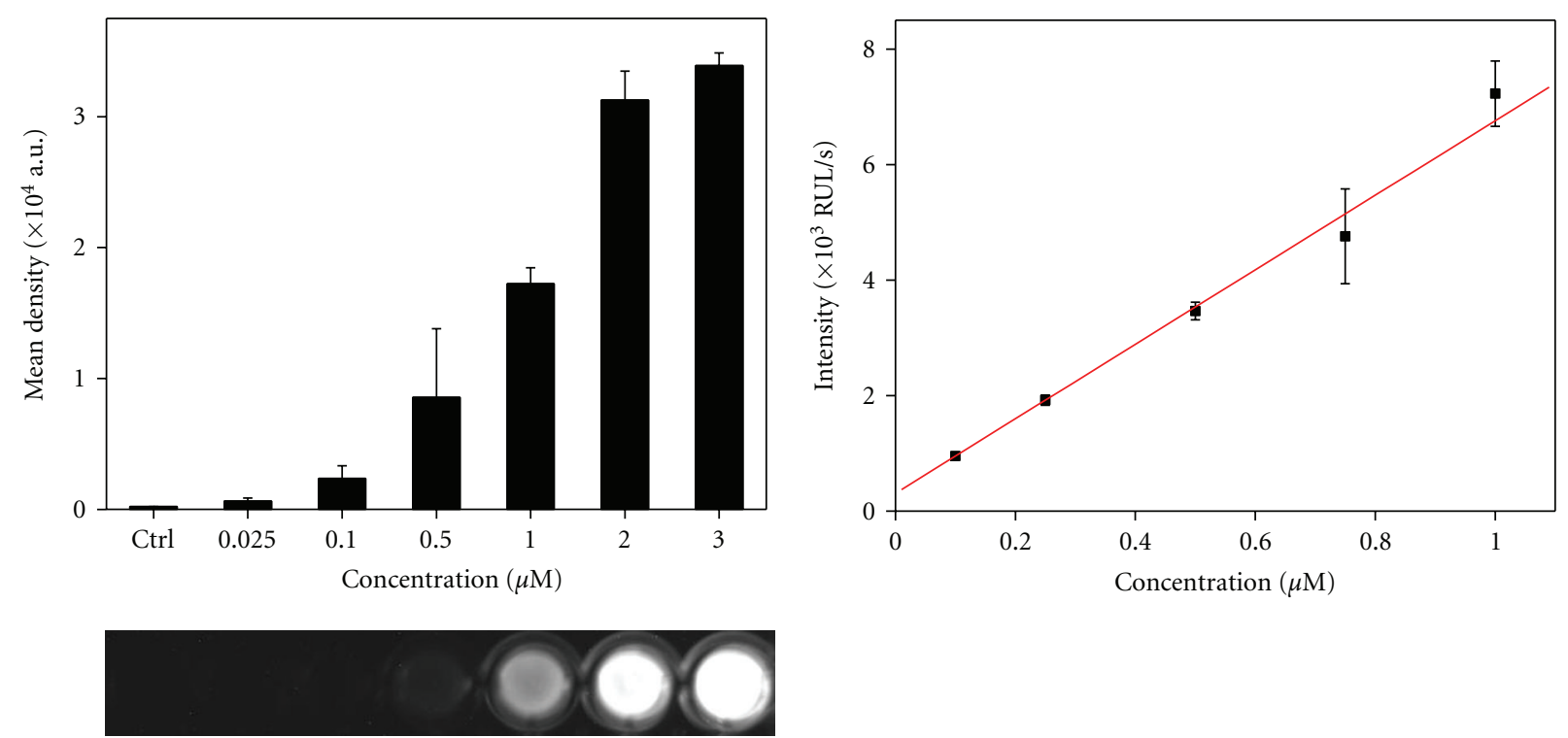

(a)

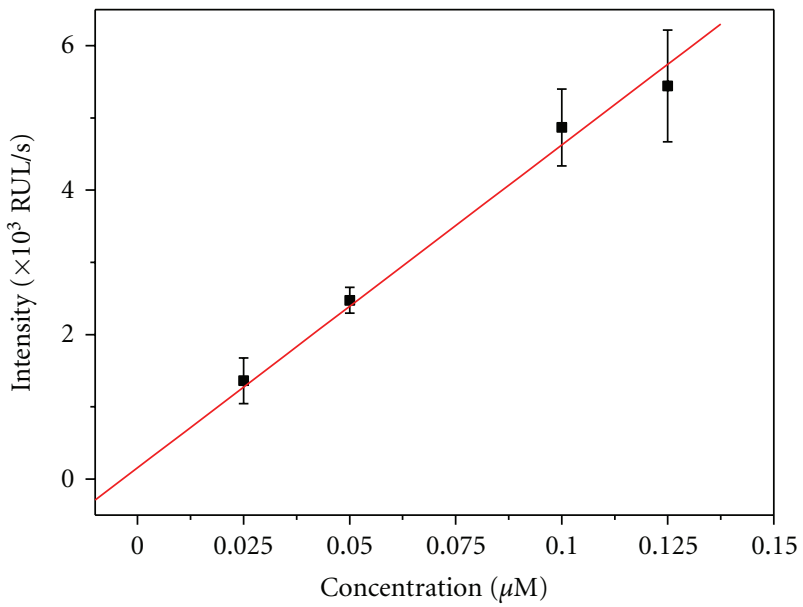

(c)

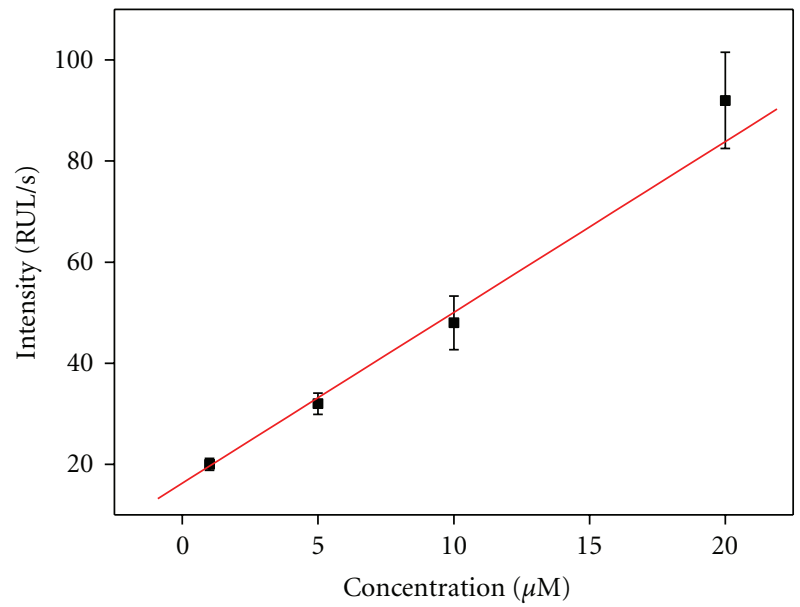

(d)

FIGURE 3: (a) Imaging of chemiluminescence produced by the POMs $\left(2.5 \mathrm{mg} / \mathrm{mL}\right.$ ) with $\mathrm{H}_{2} \mathrm{O}_{2}$ at various concentrations; (b), (c), and (d) Correlation of the POMs $(2.5 \mathrm{mg} / \mathrm{mL})$ to various concentrations of $\mathrm{H}_{2} \mathrm{O}_{2}$ in $\mathrm{PBS}$ under different operating voltage $(\mathrm{OV})$. (b) $\mathrm{OV}=-500 \mathrm{eV}$; (c) $\mathrm{OV}=-600 \mathrm{eV}$; (d) $\mathrm{OV}=-400 \mathrm{eV}$. 


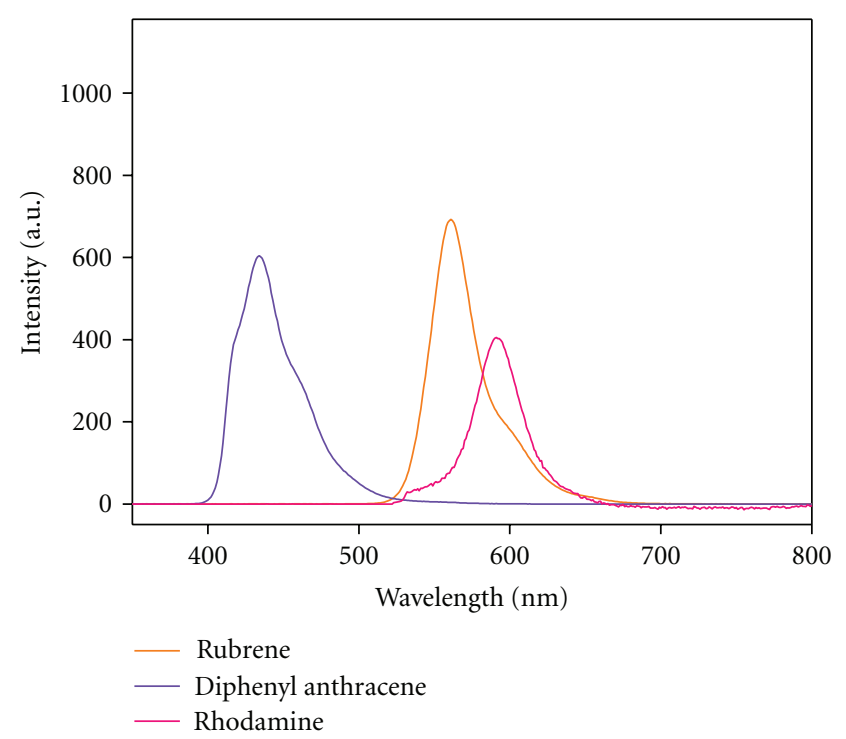

FIGURE 4: Chemiluminescence emission spectra of peroxalate ester oligomers and different dyes in dimethyl phthalate in the presence of $\mathrm{H}_{2} \mathrm{O}_{2}$.

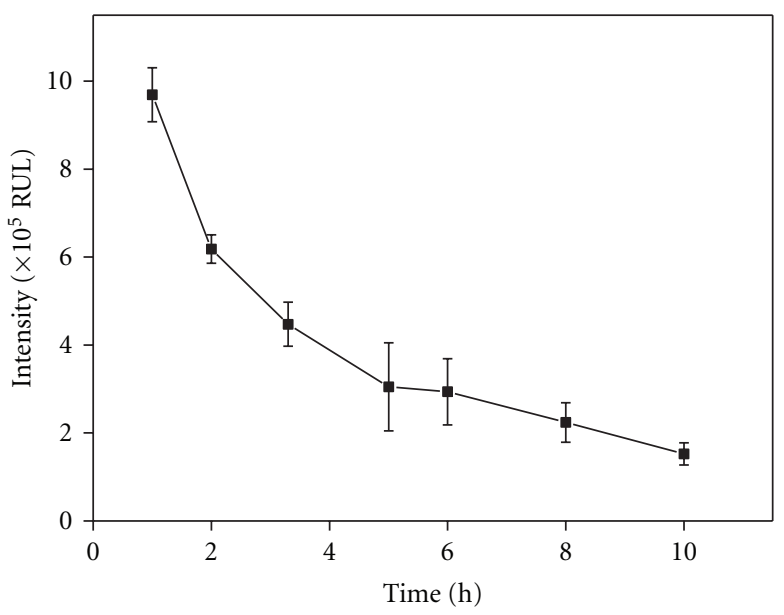

FIgUre 5: Stability of the POMs in deionized water. POMs: $2.5 \mathrm{mg} / \mathrm{mL}, \mathrm{H}_{2} \mathrm{O}_{2}: 0.2 \mu \mathrm{M}, \mathrm{OV}=-550 \mathrm{eV}$.

\section{Results and Discussion}

3.1. The Composition of the Nanomicelles. The amphiphilic copolymer PEG-PCL was chosen as the scaffold of the POMs because it is biodegradable and biocompatible that had been extensively used for drug delivery [26, 27]. The hydrophilic PEG corona should enhance the biocompatibility and stability in physiologic environments, while the hydrophobic PCL cores could stabilize encapsulated peroxalate esters from water hydrolysis. In the present work, the copolymer PEGPCL was synthesized by ring-opening polymerization, and its molecular weight was determined by GPC to be $12 \mathrm{kDa}$ with polydispersity index (PI) of 1.32. Peroxalate ester oligomer was synthesized with 4-hydroxybenzyl alcohol, 1, 8-octanediol, and oxalyl chloride. The structure of product was confirmed by FT-IR and ${ }^{1} \mathrm{H}-\mathrm{NMR}$ (Figure 1 ). In
FT-IR spectrum, the two groups of split peaks around $1732 \mathrm{~cm}^{-1} / 1688 \mathrm{~cm}^{-1}$ and $1193 \mathrm{~cm}^{-1} / 1165 \mathrm{~cm}^{-1}$ indicated the structure of mixed aromatic/aliphatic peroxalates. In ${ }^{1} \mathrm{H}$ NMR spectrum, the characteristic resonances of $7.3 \mathrm{ppm}(\mathrm{b})$, $5.4 \mathrm{ppm}$ (c), $4.3 \mathrm{ppm}$ (a), and $1.4 \mathrm{ppm}$ (d) were observed, which further identified this structure. The molecular weight of peroxalate ester oligomer determined by GPC was $600 \mathrm{Da}$ with PI of 1.21. Thus, it was clear that the oligomer synthesized had a trimer structure of peroxalate ester based on GPC result. During the preparation procedure of POMs, peroxalate ester oligomers together with fluorescent dyes could be totally encapsulated inside the hydrophobic core of PEG-PCL nanomicelles, which could protect the peroxalate from contacting the water and postpone the hydrolysis. The hydrophilic PEG segment would surround outside the POMs to stabilize these micelles in the aqueous solution.

3.2. The Size and Morphology of POMs. Peroxalate ester oligomer and the fluorescent dye (rubrene) were encapsulated in the PEG-PCL copolymer micelles forming the POMs during preparation procedure. As determined by DLS, the obtained POMs had a number-weighted hydrodynamic diameter of $119 \pm 0.9 \mathrm{~nm}$ (Figure 2(a)). The transmission electron microscopic (TEM) image manifested that these nanomicelles were spherical in outline with an average diameter of about $60 \mathrm{~nm}$ (Figure 2(b)), which is smaller than that from DLS because they were observed in the dry state. The small size of the nanomicelles should make them suitable for imaging hydrogen peroxide in cellular mitochondria because micelles in this size range had a higher opportunity for cell penetrating [28].

3.3. Chemiluminescence of POMs with Hydrogen Peroxide. These obtained POMs reacting with hydrogen peroxide produced high-energy intermediate, which could chemically excite fluorescent dye to give an extent chemiluminescence. Although its intensity was too weak to be recognized by naked eye, the image of chemiluminescence could be acquired by a low-temperature CCD camera. In Figure 3(a), the chemiluminescence image of hydrogen peroxide and POMs in a 96-well plate was shown. It was clear that with the increase of the hydrogen peroxide concentration from 0.025 to $3 \mu \mathrm{M}$ (in PBS $\mathrm{pH}=7.4$ ), the brighter wells could be seen. The quantitative analyses above the image confirmed that the higher hydrogen peroxide concentration was, the higher chemiluminescence intensity would be.

Meanwhile, the chemiluminescence intensity was linearly related to the concentrations of hydrogen peroxide. As shown in Figure 3(b), the chemiluminescence intensity increased linearly with the concentration of the hydrogen peroxide in the range of $0.1-1 \mu \mathrm{M}$, which indicated that the concentration hydrogen peroxide in the cells or organs could be quantitatively calculated according to the standard curve. Further, by changing operating voltage of luminometer, the sensitivity of the device was adjusted and adopted to cover both lower (Figure 3(c)) and higher (Figure 3(d)) concentration ranges. These results demonstrated that the POMs were quite sensitive to hydrogen peroxide and could 


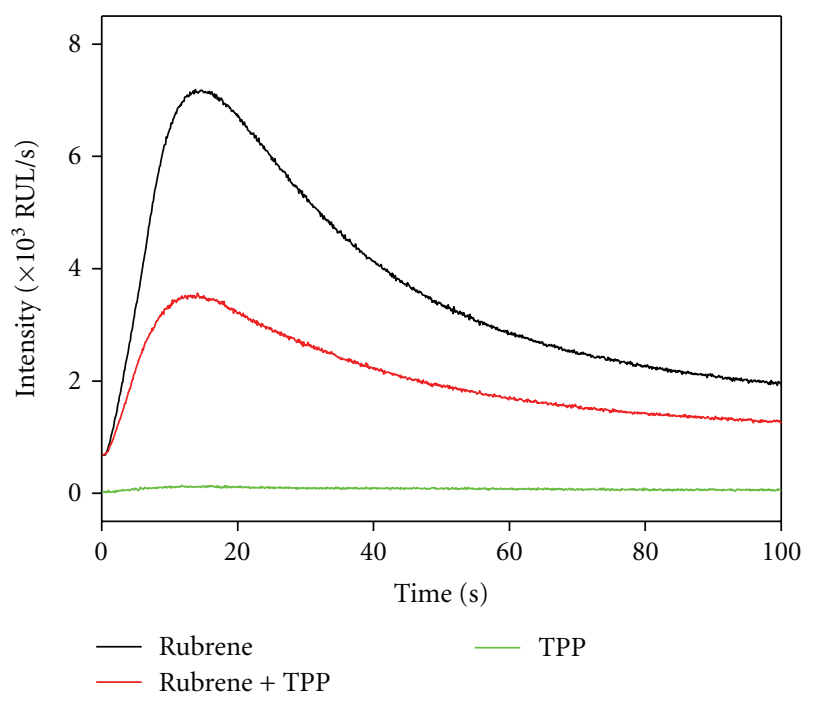

(a)

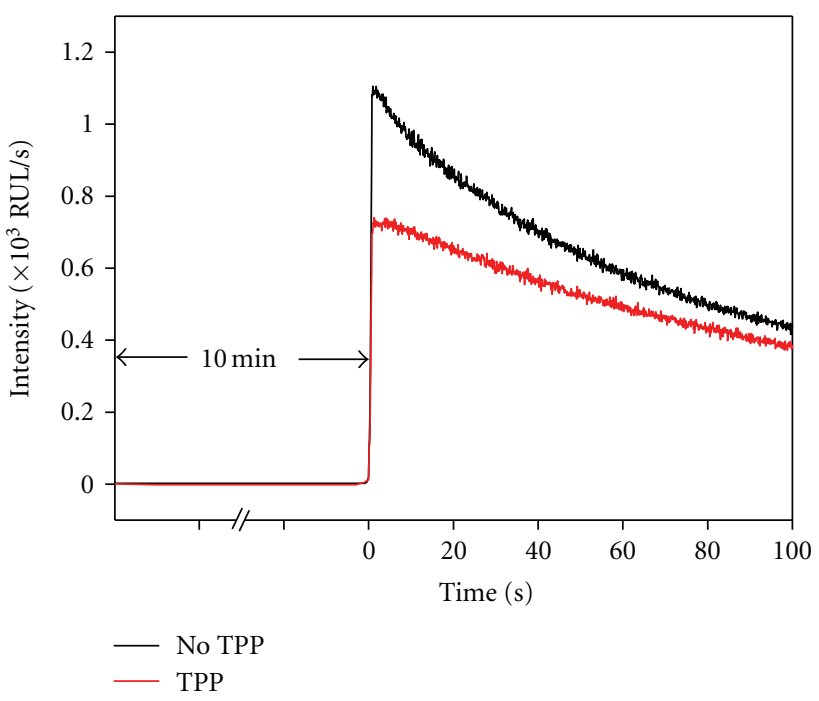

(b)

FIGURE 6: Chemiluminescence of (a) rubrene, rubrene plus TPP, and TPP-encapsulated POMs, respectively, in the presence of $0.2 \mu \mathrm{M} \mathrm{H}_{2} \mathrm{O}_{2}$; (b) no rubrene-encapsulated POMs with and without TPP in the presence of $0.2 \mu \mathrm{M} \mathrm{H}_{2} \mathrm{O}_{2}$, and the addition rubrene ( $\left.0.2 \mathrm{mg}\right)$ after $10 \mathrm{~min}$. All concentrations of POMs were $2.5 \mathrm{mg} / \mathrm{mL}, \mathrm{OV}=-550 \mathrm{eV}$.

be used to detect physiological concentration of hydrogen peroxide.

The chemical reaction between peroxalate and hydrogen peroxide formed an intermediate 1,2-dioxetanedione which was potential to excite a variety of fluorescent dyes, suitable for a particular application. The tunable wavelength of peroxalate chemiluminescence system was investigated by encapsulating different dyes in the POMs and measuring their emission spectra. Figure 4 demonstrated that peroxalate esters oligomers could excite diphenyl anthracene, rubrene, and rhodamine in the presence of hydrogen peroxide, producing fluorescence at 430,560, and $600 \mathrm{~nm}$, respectively. The chemiluminescence emission wavelength could be readily tuned to near infrared region by using a near-infrared fluorescent dye and therefore this method can be used potentially in deep tissue imaging owing to the minimal tissue scattering and absorption to the near infrared [29].

Although the POMs have been proved a good carrier to detect the $\mathrm{H}_{2} \mathrm{O}_{2}$ in the aqueous solution, the most challenging matter of this system is the instability of peroxalate against water because of a fast hydrolysis in aqueous solutions which makes them unsuitable for physiological applications. However, the encapsulation of peroxalate esters oligomers inside the PEG-PCL micelles could protect them from water hydrolysis by providing a hydrophobic environment with low water permeability [30]. Hence, we investigated the stability of these POMs by incubating them in water for different time and measuring their chemiluminescence in the presence of hydrogen peroxide. Figure 5 demonstrated the chemiluminescence intensity from the reaction between peroxalate esters, and $\mathrm{H}_{2} \mathrm{O}_{2}$ decreased as the incubation time in water extended. The POMs had a half-life longer than 2 hours, suggesting that the POMs effectively prevented the peroxalate esters from water hydrolysis, compared to an analogous work with a half-life of $30 \mathrm{~min}$ [16]. It suggested that the polymer nanomicelles effectively improved the stability of the peroxalate esters. The improved stability of the peroxalate ester oligomers should allow them to image hydrogen peroxide in physiological condition.

3.4. Characterization of the TPP-Encapsulated POMs. The photodynamic therapy agent (mesotetraphenylporphine, TPP) was encapsulated in POMs using the same method as described above. And the size of TPP-encapsulated POMs is to have similar average size with no TPP-encapsulated POMs by DLS measurement. As shown in Figure 6(a), the TPPencapsulated POMs did not have detectable luminescence because the high-energy intermediate could not directly transfer chemical energy to TPP molecule. Although POMs could not directly excite TPP, it could stimulate rubrene for a remarkable chemiluminescence. Meanwhile, with the same concentration of POMs and rubrene, the addition of TPP weakens the fluorescence of rubrene dye, which indicated that part of energy had transferred to TPP. Rubrene played as an effective switchover for transferring chemical energy to photon energy.

In order to further verify whether TPP has absorbed the fluorescence produced by rubrene, a subtle designed experiment was carried on. According to the work reported [10], in the absence of fluorescence dyes, the high-energy intermediate dioxetanedione can maintain its energy for a while until being exposed to the dyes. In this experiment, firstly, only POMs with and without TPP were incubated in the presence of $0.2 \mu \mathrm{M} \mathrm{H}_{2} \mathrm{O}_{2}$. No luminescence was observed in these systems as displayed in Figure 6(b). Both of these two systems had no rubrene inside them. After 10 minutes, when $0.2 \mathrm{mg}$ rubrene was added into these two kinds of POMs, respectively, significant chemiluminescence signals 


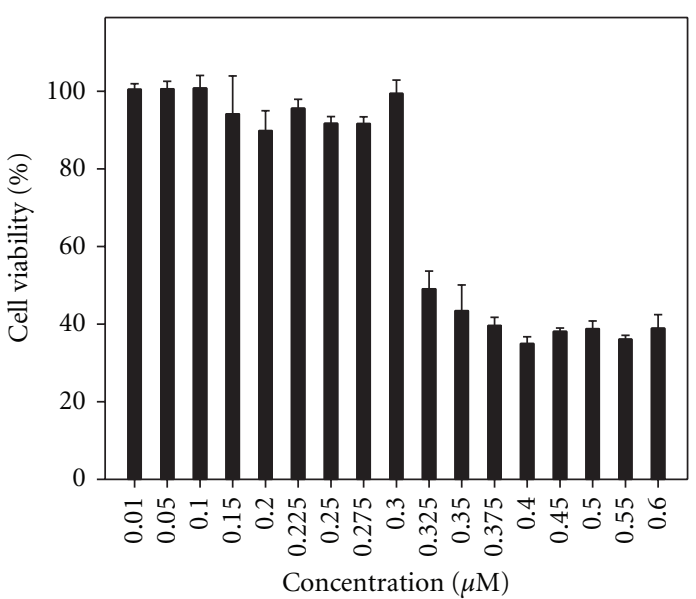

(a)

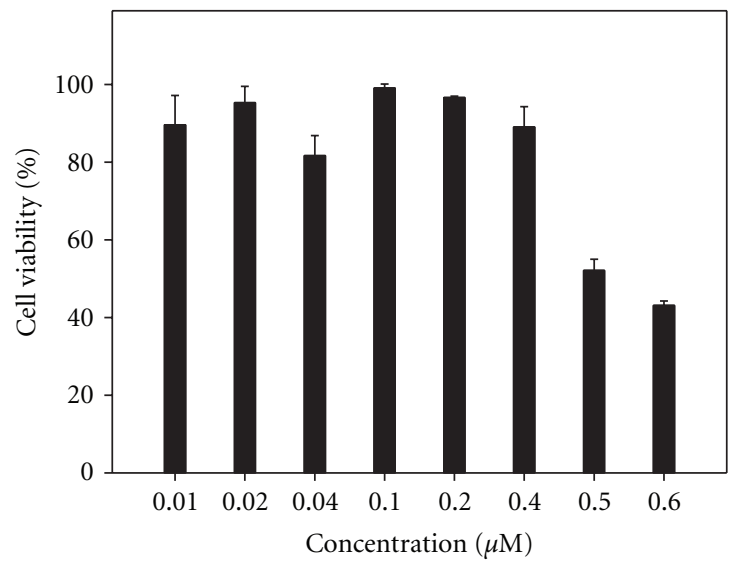

(b)

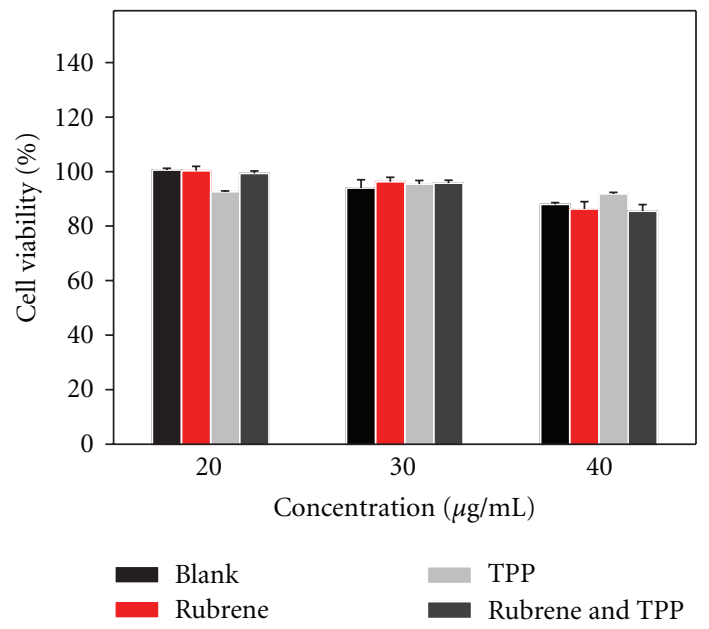

(c)

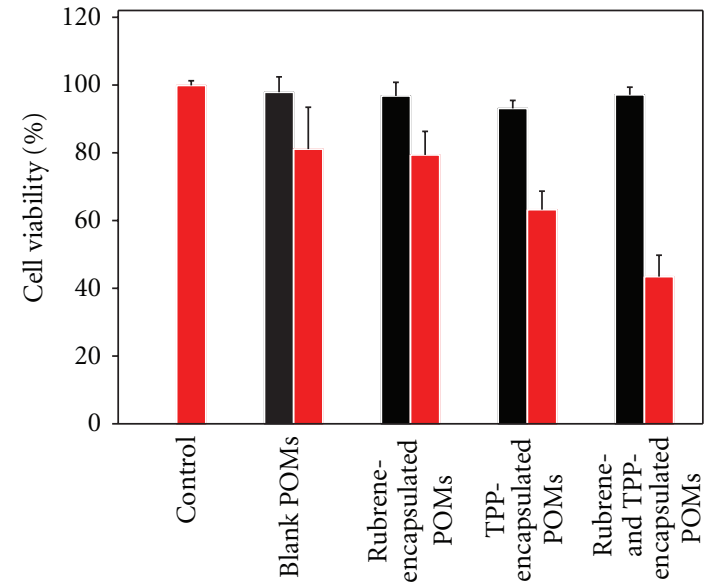

$-\mathrm{H}_{2} \mathrm{O}_{2}$

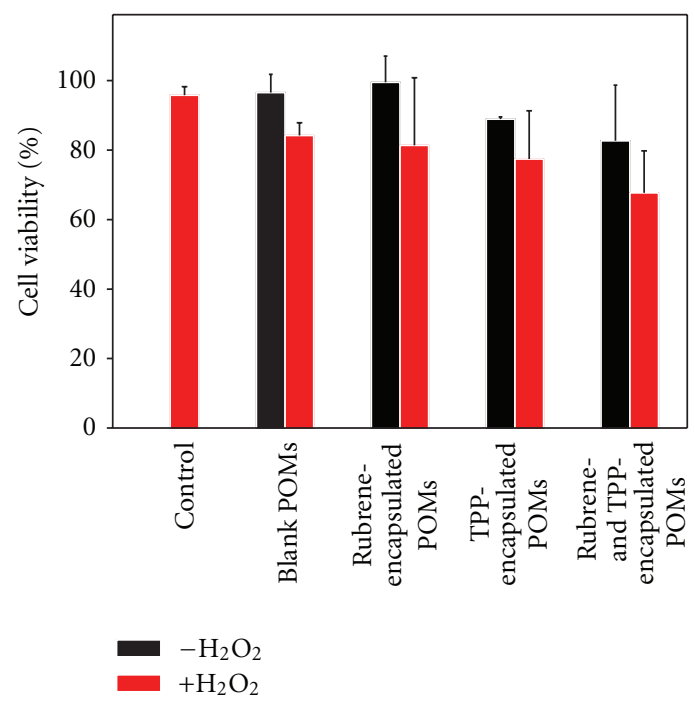

(e)

Figure 7: The cytotoxicity of $\mathrm{H}_{2} \mathrm{O}_{2}$ to (a) $\mathrm{C} 6$ and (b) LoVo cell lines at various concentrations in PBS (pH = 7.4); (c) the cytotoxicity of a diversity of POMs to $\mathrm{C} 6$ cell lines without $\mathrm{H}_{2} \mathrm{O}_{2}$. The cytotoxicity of a diversity of POMs to (d) C6 cells and (e) LoVo cells in the presence of $0.2 \mu \mathrm{M} \mathrm{H}_{2} \mathrm{O}_{2}$ or not, control means only $\mathrm{H}_{2} \mathrm{O}_{2}$ treated. All concentrations of POMs were $20 \mu \mathrm{g} / \mathrm{mL}$, Rubrene $0.3 \mu \mathrm{g} / \mathrm{mL}$, and TPP $2 \mu \mathrm{g} / \mathrm{mL}$. Error bars indicate standard deviations from $\geq 3$ measurements. 
were observed in the systems. However, the POMs encapsulating TPP showed a reduced chemiluminescence signal compared to the blank POMs, which clearly supporting that TPP absorbed part of the energy. Therefore, part of the chemical energy from the reaction between POMs and $\mathrm{H}_{2} \mathrm{O}_{2}$ could be transformed to light energy with the help of rubrene, which indicated that the POMs and rubrene played the role of a laser light in the presence of $\mathrm{H}_{2} \mathrm{O}_{2}$. Even the chemiluminescence intensity was quite low compared with a real laser source, this system opened a new window in the PDT field because no additional laser light was needed in this system. For the traditional PDT system, external laser light was needed to excite the PPT in the tumor treatment procedure. However, restricted by its penetration depth of the laser light, only the tumor close to the skin could be killed effectively. In our system, The TPP drug was excited by the inner chemiluminescence, which avoided the use of external laser resource, consequently overcoming the problem of the depth of the tumor site. Furthermore, particles with nanosize had been proved to be accumulated in the tumor site by the EPR effect or targeting strategy [31]. So the TPP-loaded POMs may greatly improve the therapy effect compared to the traditional PDT method.

3.5. The Anticancer Properties of the TPP-Encapsulated POMs. The chemiluminescence radiation produced by the POMs in the presence of hydrogen peroxide could act as an inner light source to excite PDT drug to play curative effect. To verify the antitumor effect of the TPP-encapsulated POMs, in vitro cytotoxicity tests of the TPP-loaded POMs against C6 and LoVo cell lines were conducted. As well known, high concentration of hydrogen peroxide was cytotoxic to cells. The control experiment of cells coincubated with different concentration of hydrogen peroxide was performed, and their cytotoxicities were also evaluated by MTT assay. The results clearly showed that the critical cytotoxic concentration of hydrogen peroxide is $0.3 \mu \mathrm{M}$ for C6 cells (Figure 7(a)) and $0.4 \mu \mathrm{M}$ for LoVo cells (Figure $7(\mathrm{~b})$ ), respectively, helping us to choose a safe $\mathrm{H}_{2} \mathrm{O}_{2}$ concentration which was nontoxic to cells but could acquire maximum chemiluminescence. Therefore, hydrogen peroxide concentration $0.2 \mu \mathrm{M}$ was chosen for all the cell cytotoxicity experiments. The cytotoxicity of different kinds of POMs (e.g., with or without rubrene) was checked without hydrogen peroxide treatment (Figure 7(c)). No obvious cytotoxicity was observed at low concentration except a slight cytotoxicity at high concentration. So it seemed safe to conclude that the blank POMs have no or little cytotoxicity against the test cells. Therefore, the POMs used in the following experiment were fixed on the concentration of $20 \mu \mathrm{g} / \mathrm{mL}$, and the hydrogen peroxide was the same concentration of $0.2 \mu \mathrm{M}$. For C6 cells, when they were treated with the POMs, in the absence of hydrogen peroxide, no or little cytotoxicity was observed in all the samples as shown in Figure 7(d). However, when hydrogen peroxide was added into the wells, an increasing cytotoxicity is observed. Furthermore, C6 cells treated with the POMs having both rubrene and TPP showed lowest cell viability compared with other groups, indicating TPP and rubrene having a combined antitumor effect, which might resulted from the chemiluminescence-stimulated PDT effect of TPP. Similar results were also observed in the LoVo cell line cytotoxicity experiment (Figure 7(e)).

\section{Conclusion}

Chemiluminescent POMs were successfully developed to image tumor signal molecules: hydrogen peroxide. These POMs could improve the stability of peroxalates in aqueous system and were sensitive to low concentration of hydrogen peroxide within the physiological range. According to the linear correlation between chemiluminescent intensity and the concentration of hydrogen peroxide, a facile and robust approach for monitoring hydrogen peroxide molecules was established, which should be helpful to clinical diagnosis. When PDT drug TPP was encapsulated inside the POMs together with rubrene, the TPP-encapsulated POMs performed an anticancer property in the presence of hydrogen peroxide which was stimulated by self-produced chemiluminescence not an external laser. This system enabled us to open a new window in the PDT field without using external laser light resource to treat the malignant tumor.

\section{Acknowledgment}

This work has been supported by the Natural Science Foundation of China (nos. 21074051, 51033002, and 20874042).

\section{References}

[1] K. Rutault, C. Alderman, B. M. Chain et al., "Reactive oxygen species active human peripheral blood denditic cells," Free Radialc Biology and Medicine, vol. 26, no. 1-2, pp. 232-238, 1999.

[2] S. Dominici, A. Visvikis, L. Pieri et al., "Redox modulation of NF- $\kappa \mathrm{B}$ nuclear translocation and DNA binding in metastatic melanoma. The role of endogenous and $\gamma$-glutamyl transferase-dependent oxidative stress," Tumori, vol. 89, no. 4, pp. 426-433, 2003.

[3] S. G. Rhee, " $\mathrm{H}_{2} \mathrm{O}_{2}$, a necessary evil for cell signaling," Science, vol. 312, no. 5782, pp. 1882-1883, 2006.

[4] M. Giorgio, M. Trinei, E. Migliaccio, and P. G. Pelicci, "Hydrogen peroxide: a metabolic by-product or a common mediator of ageing signals?" Nature Reviews Molecular Cell Biology, vol. 8, no. 9, pp. 722A-728A, 2007.

[5] H. Ohshima, M. Tatemichi, and T. Sawa, "Chemical basis of inflammation-induced carcinogenesis," Archives of Biochemistry and Biophysics, vol. 417, no. 1, pp. 3-11, 2003.

[6] E. W. Miller, A. E. Albers, A. Pralle, E. Y. Isacoff, and C. J. Chang, "Boronate-based fluorescent probes for imaging cellular hydrogen peroxide," Journal of the American Chemical Society, vol. 127, no. 47, pp. 16652-16659, 2005.

[7] M. C. Y. Chang, A. Pralle, E. Y. Isacoff, and C. J. Chang, "A selective, cell-permeable optical probe for hydrogen peroxide in living cells," Journal of the American Chemical Society, vol. 126, no. 47, pp. 15392-15393, 2004.

[8] A. E. Albers, V. S. Okreglak, and C. J. Chang, "A FRET-based approach to ratiometric fluorescence detection of hydrogen peroxide," Journal of the American Chemical Society, vol. 128, no. 30, pp. 9640-9641, 2006. 
[9] R. W. Tsien and R. Y. Tsien, "Calcium channels, stores, and oscillations," Annual Review of Cell Biology, vol. 6, pp. 715760, 1990.

[10] M. M. Rauhut, L. J. Bollyky, B. G. Roberts et al., "Chemiluminescence from reactions of electronegatively substituted aryl oxalates with hydrogen peroxide and fluorescent compounds," Journal of the American Chemical Society, vol. 89, no. 25, pp. 6515-6522, 1967.

[11] C. V. Stevani, S. M. Silva, and W. J. Baader, "Studies on the mechanism of the excitation step in peroxyoxalate chemiluminescence," European Journal of Organic Chemistry, no. 24, pp. 4037-4046, 2000.

[12] S. M. Silva, K. Wagner, D. Weiss, R. Beckert, C. V. Stevani, and W. J. Baader, "Studies on the chemiexcitation step in peroxyoxalate chemiluminescence using steroid-substituted activators," Luminescence, vol. 17, no. 6, pp. 362-369, 2002.

[13] D. Lee, S. Khaja, J. C. Velasquez-Castano et al., "In vivo imaging of hydrogen peroxide with chemiluminescent nanoparticles," Nature Materials, vol. 6, no. 10, pp. 765-769, 2007.

[14] L. Liu and R. P. Mason, "Imaging $\beta$-galactosidase activity in human tumor xenografts and transgenic mice using a chemiluminescent substrate," PLOS ONE, vol. 5, no. 8, Article ID e12024, 2010.

[15] S. Kim, K. Seong, O. Kim et al., "Polyoxalate nanoparticles as a biodegradable and biocompatible drug delivery vehicle," Biomacromolecules, vol. 11, no. 3, pp. 555-560, 2010.

[16] M. Dasari, D. Lee, V. R. Erigala, and N. Murthy, "Chemiluminescent PEG-PCL micelles for imaging hydrogen peroxide," Journal of Biomedical Materials Research-Part A, vol. 89, no. 3, pp. 561-566, 2009.

[17] R. B. Thompson and S. E. Shaw, "Peroxyoxalate chemiluminescence in microemulsions," Langmuir, vol. 4, no. 1, pp. 106110,1988

[18] A. Kamyshny and S. Magdassi, "Chemiluminescence immunoassay in microemulsions," Colloids and Surfaces B: Biointerfaces, vol. 11, no. 5, pp. 249-254, 1998.

[19] B. C. Wilson and M. S. Patterson, "The physics, biophysics and technology of photodynamic therapy," Physics in Medicine and Biology, vol. 53, no. 9, pp. R61-R109, 2008.

[20] Y. N. Konan, R. Gurny, and E. Allémann, "State of the art in the delivery of photosensitizers for photodynamic therapy," Journal of Photochemistry and Photobiology B: Biology, vol. 66, no. 2, pp. 89-106, 2002.

[21] L. O. Cinteza, T. Y. Ohulchanskyy, Y. Sahoo, E. J. Bergey, R. K. Pandey, and P. N. Prasad, "Diacyllipid micelle-based nanocarrier for magnetically guided delivery of drugs in photodynamic therapy," Molecular Pharmaceutics, vol. 3, no. 4, pp. 415-423, 2006.

[22] A. Roby, S. Erdogan, and V. P. Torchilin, "Solubilization of poorly soluble PDT agent, meso-tetraphenylporphin, in plain or immunotargeted PEG-PE micelles results in dramatically improved cancer cell killing in vitro," European Journal of Pharmaceutics and Biopharmaceutics, vol. 62, no. 3, pp. 235240, 2006.

[23] S. Kim, T. Y. Ohulchanskyy, H. E. Pudavar, R. K. Pandey, and P. N. Prasad, "Organically modified silica nanoparticles co-encapsulating photosensitizing drug and aggregationenhanced two-photon absorbing fluorescent dye aggregates for two-photon photodynamic therapy," Journal of the American Chemical Society, vol. 129, no. 9, pp. 2669-2675, 2007.

[24] S. Kim, H. Huang, H. E. Pudavar, Y. Cui, and P. N. Prasad, "Intraparticle energy transfer and fluorescence photoconversion in nanoparticles: an optical highlighter nanoprobe for two-photon bioimaging," Chemistry of Materials, vol. 19, no. 23 , pp. 5650-5656, 2007.

[25] S. Zhuang, J. T. Demirs, and I. E. Kochevar, "p38 mitogenactivated protein kinase mediates bid cleavage, mitochondrial dysfunction, and caspase- 3 activation during apoptosis induced by singlet oxygen but not by hydrogen peroxide," Journal of Biological Chemistry, vol. 275, no. 34, pp. 2593925948, 2000.

[26] M. L. Forrest, C. Y. Won, A. W. Malick, and G. S. Kwon, "In vitro release of the mTOR inhibitor rapamycin from poly(ethylene glycol)-b-poly( $\varepsilon$-caprolactone) micelles," Journal of Controlled Release, vol. 110, no. 2, pp. 370-377, 2006.

[27] H. M. Aliabadi, A. Mahmud, A. D. Sharifabadi, and A. Lavasanifar, "Micelles of methoxy poly(ethylene oxide)-bpoly ( $\varepsilon$-caprolactone) as vehicles for the solubilization and controlled delivery of cyclosporine A," Journal of Controlled Release, vol. 104, no. 2, pp. 301-311, 2005.

[28] V. P. Torchilin, "PEG-based micelles as carriers of contrast agents for different imaging modalities," Advanced Drug Delivery Reviews, vol. 54, no. 2, pp. 235-252, 2002.

[29] C.-K. Lim, Y.-D. Lee, J. Na et al., "Chemiluminescencegenerating nanoreactor formulation for near-infrared imaging of hydrogen peroxide and glucose level in vivo," Advanced Functional Materials, vol. 20, no. 16, pp. 2644-2648, 2010.

[30] J. M. Lin and M. Yamada, "Microheterogeneous systems of micelles and microemulsions as reaction media in chemiluminescent analysis," Trends in Analytical Chemistry, vol. 22, no. 2, pp. 99-107, 2003.

[31] H. Maeda, J. Wu, T. Sawa, Y. Matsumura, and K. Hori, “Tumor vascular permeability and the EPR effect in macromolecular therapeutics: a review," Journal of Controlled Release, vol. 65, no. 1-2, pp. 271-284, 2000. 


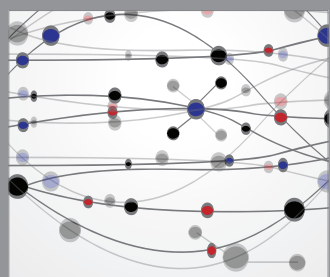

The Scientific World Journal
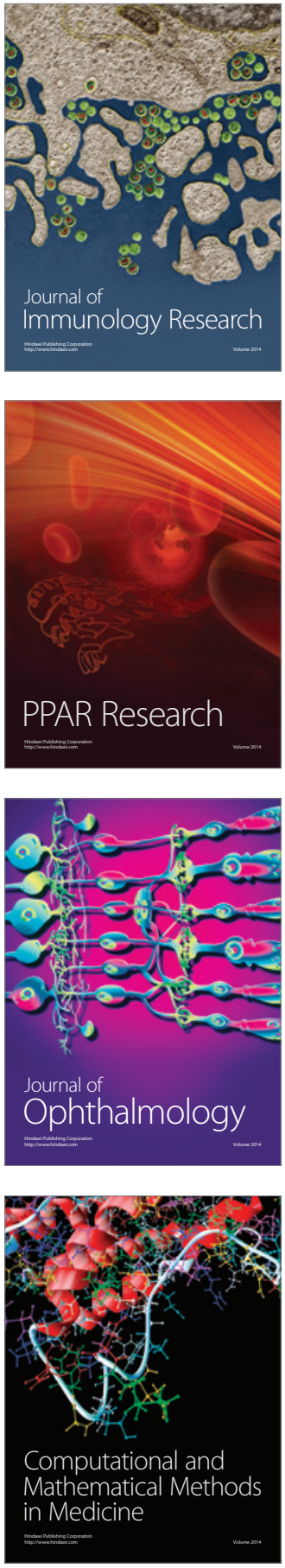

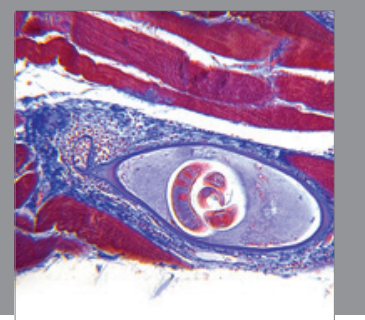

Gastroenterology

Research and Practice
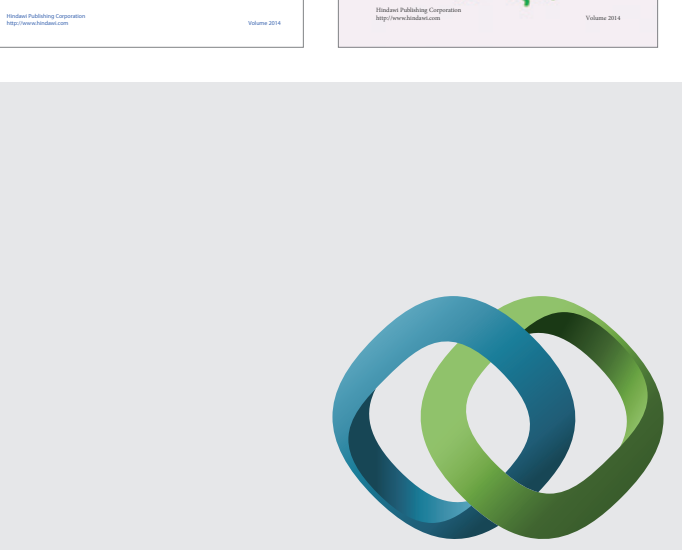

\section{Hindawi}

Submit your manuscripts at

http://www.hindawi.com
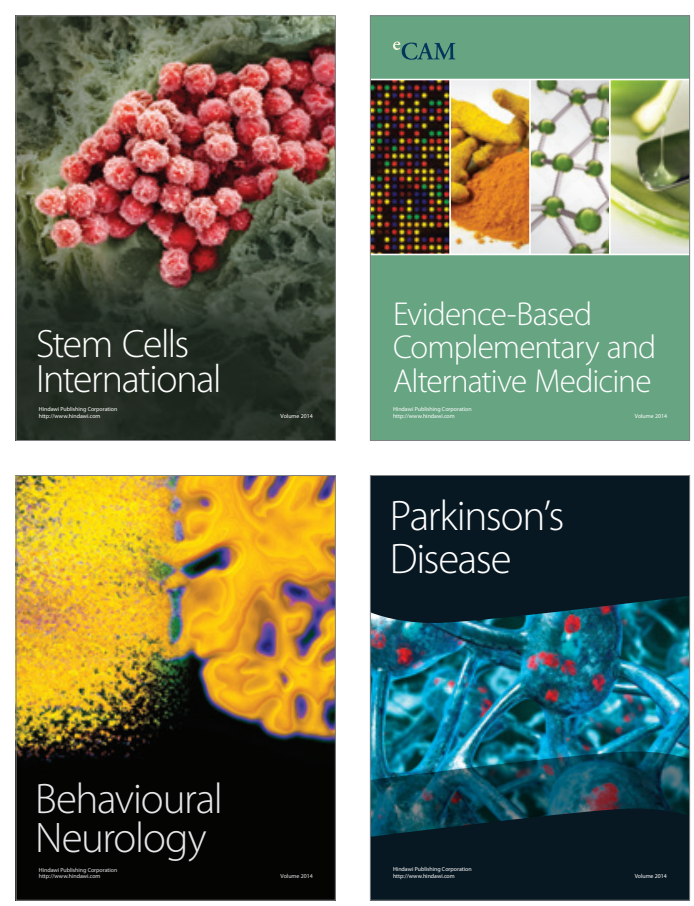

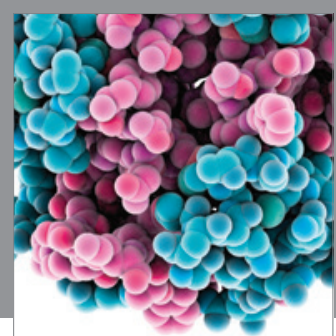

Journal of
Diabetes Research

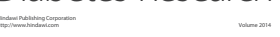

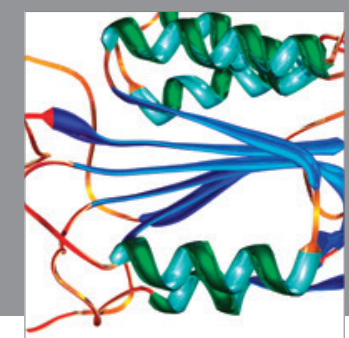

Disease Markers
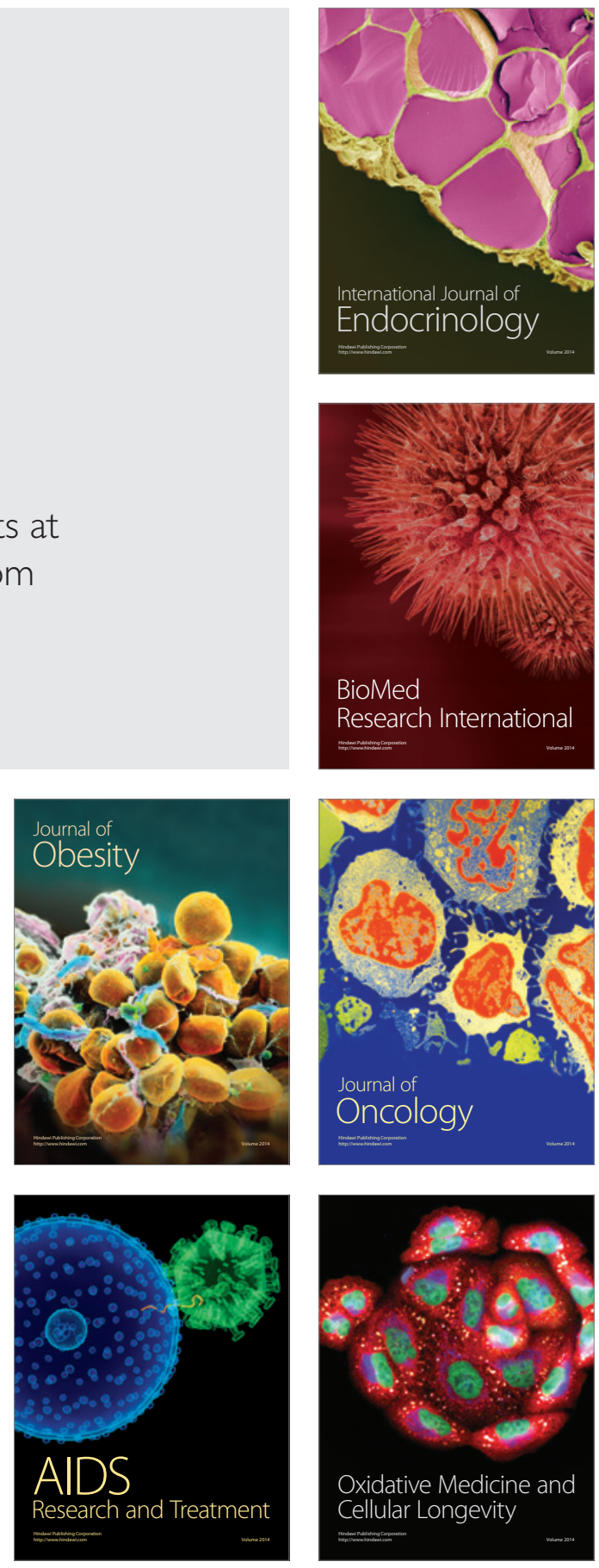